Article

\title{
Strain Energy Effects in the Spinodal Decomposition of $\mathrm{Cu}-\mathrm{Ni}(\mathrm{Fe})$ Nanolaminate Coatings
}

\author{
Alan F. Jankowski \\ Department of Mechanical Engineering, Edward E. Whitacre Jr. College of Engineering, Texas Tech \\ University, Lubbock, TX 79409-1021, USA; E-Mail: alan.jankowski@ttu.edu; \\ Tel.: +1-806-834-5203; Fax: +1-806-742-3540 \\ Academic Editor: Alessandro Lavacchi
}

Received: 9 May 2015 / Accepted: 16 June 2015 / Published: 26 June 2015

\begin{abstract}
A model for spinodal decomposition must account for interface effects that include gradient and strain energy terms. The measurement of diffusion in the $\mathrm{Cu}-\mathrm{Ni}(\mathrm{Fe})$ alloy for the special case of nanolaminate structured coatings is considered wherein the composition fluctuation is one-dimensional along $<111>$. An analytic approach is taken to model the kinetics of the transformation process that provides quantification of the strain energy dependence on the composition wavelength, as well as the intrinsic diffusivities and higher-order gradient-energy coefficients. The variation of the wave amplification factor $R$ with wavenumber is modeled first to incorporate the boundary condition for growth at infinite wavelength. These results are used next to determine the gradient energy coefficients

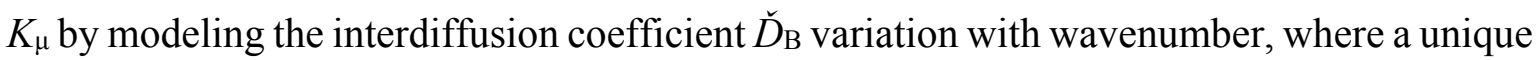
determination of the diffusion coefficient $\check{D}$ is made. The value of the strain energy component that originates from interface strains associated with the epitaxial growth between layers is then determined by assessing the variation of wavelength-dependent amplification factors. A peak value of $9.4 \times 10^{7} \mathrm{~J} \cdot \mathrm{m}^{-3}$ for the strain energy is computed for $\mathrm{Cu}-\mathrm{Ni}(\mathrm{Fe})$ nanolaminate coatings with $2-4 \mathrm{~nm}$ composition wavelengths.
\end{abstract}

Keywords: nanolaminates; spinodal; strain energy; diffusion

\section{Introduction}

The diffusional transformation of spinodal decomposition [1-10] proceeds by the growth of a periodic composition fluctuation as the $\alpha$-phase matrix decomposes at temperatures within the spinodal into $\alpha^{\prime}$ 
and $\alpha^{\prime \prime}$ phases, without a change in the crystal structure. The evolution continues of the linear Cahn-Hilliard model for spinodal decomposition. The effect of nonlinear relaxation dynamics [11] is considered with respect to the interaction of phase boundaries and their motion. The case for multilayered structures is investigated wherein it can be modeled that the motion of an interface is affected by interactions with second nearest interfaces, leading towards the tendency of doubling the composition wavelength over time. Mossbauer measurements [12] evidence the continued decomposition of $\mathrm{Cu}-\mathrm{Ni}(\mathrm{Fe})$ at room temperature.

The relevance of lattice coherency effects are seen in material behaviors of $\mathrm{Cu}-\mathrm{Ni}(\mathrm{Fe})$ alloys including mechanical and magnetic. Numerous spinodal decomposition studies use the processing of bulk alloys. Mechanical alloying [13] provides a route to produce nanostructured powder precursors as well. The effect of coherency strains is evident in the yield strength [14,15] of age-hardened alloys where maximum strength was achieved for lower aging temperatures. An advantage of spinodal decomposition for mechanical hardening [16] is that excellent dimensional stability can be maintained since no crystallographic changes take place. A giant magnetoresistance behavior was introduced [17] by aging a bulk alloy through processing via spinodal decomposition with uniaxial deformation to produce local superlattice-type nanostructures. Coatings [18] on low-carbon steels have been evaluated for improved corrosion behavior. In multilayered form, a more recent investigation [19] of ferromagnetic nanostructured multilayers considers the effect of growth conditions to superparamagnetic contributions to magnetoresistance behavior that result from composition-rich regions.

This study considers the effects of strain energy from a one-dimensional modulation in nanolaminate structures on the kinetics of the spinodal decomposition process.

\section{Background}

The free energy $f$ at a temperature $T_{\mathrm{i}}$ is considered for an alloy system composed of elements $\mathrm{A}$ and B. The compositions $c^{\prime}$ and $c_{\alpha^{\prime \prime}}$ indicate the lower and upper bounds, respectively, for the composition $c$ B of element B within the miscibility gap. The local minima in the free energy curve, i.e., at $f^{\prime}=\partial f / \partial c=0$, provide the locus of points at temperatures $T_{\mathrm{i}}$ that form the miscibility gap.

The phase transformation process of spinodal decomposition [1-10] proceeds via uphill diffusion when the second-order derivative of the Helmholtz free energy $f$ with respect to composition $c$ is less than zero, i.e., $f^{\prime \prime}=\partial^{2} f / \partial c^{2}<0$. The locus of points for those compositions at temperatures that are between the bounds defined by $f^{\prime \prime}=0$ is the chemical spinodal. There is no barrier for decomposition for temperatures within the spinodal $T_{\mathrm{i}}<T_{\mathrm{s}}$. Decomposition for one-dimensional diffusion is found [20] where satellite reflections are observed about the Bragg peaks in X-ray diffraction patterns for a heat treated $\mathrm{Cu}-\mathrm{Ni}-\mathrm{Fe}$ alloy. An initial explanation considers an interfacial energy term [1] between adjacent atomic planes. The additional consideration of a gradient energy term $[3,4]$ provides the identification of a critical wavenumber $\beta_{c}$ to the composition fluctuation where the wavenumber-dependent interdiffusivity $\check{D}_{\mathrm{B}}$ equals zero. The decomposition stops and decay occurs beyond a critical wavenumber, i.e., $\beta>\beta_{\mathrm{c}}$, where maximum growth occurs at a wavenumber $\beta_{\mathrm{m}}$ (classically, equal to $\beta_{\mathrm{c}} / \sqrt{2}$ ).

The presence of coherency effects [5] from a strained lattice will further depress the transformation temperature of the chemical spinodal $T_{\mathrm{s}}$ to a coherent spinodal $T_{\mathrm{s}}{ }^{*}$. The decrease of the spinodal temperature, i.e., $T_{\mathrm{s}}-T_{\mathrm{s}}{ }^{*}$, can be significant [21] for some alloy systems such as Au-Ni with a large lattice misfit, whereas it is potentially smaller for bulk alloy systems such as $\mathrm{Cu}-\mathrm{Ni}(\mathrm{Fe})$ that have a 
smaller misfit strain. Along with a composition gradient energy, the presence of interfaces provides a strain energy term that evidences maximum growth of a characteristic composition wavelength along elastically soft directions. Consequently, an applied stress [8] during aging affects the evolving morphology of the composition fluctuation.

The linear theory of diffusion for spinodal decomposition [1-5,8,21-24] provides for the accommodation of energy associated with new evolving surfaces and interface strain for the composition fluctuations. The wavenumber dependent interdiffusivity $\check{D}_{\mathrm{B}}$ is linearly proportional to the square of the wavenumber $\beta^{2}$ where the amplification factor $R$ of growth to the composition wave equals $-\beta^{2} \cdot \check{D}_{\text {B. }}$ The values of $\beta_{\mathrm{c}}$ and $\beta_{\mathrm{m}}$ will change with the depression of the coherent spinodal temperature for the aging process.

The consideration of additional gradient-energy terms $K_{\mu}$ as (e.g., from the effects of distant interface interactions) is used in a further modified formulation [25] of diffusion. In concept, this allows for fitting a higher-order, non-quadratic relationship between the interdiffusivity $\check{D}_{\text {B }}$ and wavenumber, i.e., as can be directionally quantified using the dispersion relationship $B$. This has been accomplished by simultaneous determination $[25,26]$ of $K_{\mu}$ and $\check{D}$. However, uncertainty is introduced without direct use of the long-wavelength boundary condition.

A condition arises during decomposition that the presence of strain energy will always decrease the amplification factor $R$. The effect is to increase the magnitude of interdiffusivity $\check{D}_{\text {B }}$ outside the spinodal, but decrease its magnitude within the spinodal. This requires use of higher-order terms in a polynomial expansion that should meet the requirement for establishing an upper bound to the amplification factor variation with the dispersion relation and, consequently, a lower bound for the value of measured diffusion behavior.

The magnitude of the $2 \eta^{2} \cdot Y$ (the elastic modulus $Y$ ) strain energy depends upon the accommodation of strain $\eta$ within the crystal structure, and its variation with the dispersion relation. As the wavenumber, i.e., dispersion relation, decreases towards zero the strain through the nanolaminate structure will decay in magnitude. This represents the conditions for a bulk-diffusion couple. Maximum strain is often seen experimentally in metallic superlattices as $\mathrm{Cu}-\mathrm{Ni}(\mathrm{Fe})$ at relatively small composition wavelengths of $2-3 \mathrm{~nm}$ that correspond with a decrease in wavenumber to $3-2 \mathrm{~nm}^{-1}$. Therefore, a variation in the $2 \eta^{2} \cdot Y$ term with composition wavelength is anticipated.

\section{Experimental Section}

Nanolaminate structures consist of an alternating sequence of constituent components that form a composition fluctuation. Nanolaminate thin films of $\mathrm{Cu}_{0.53} / \mathrm{Ni}_{0.40} \mathrm{Fe}_{0.07}$ are prepared [26] by alternating the vapor flux between $\mathrm{Cu}$ and Ni-Fe sources onto a heated substrate. The deposition chamber is evacuated to a base pressure of $20 \mu \mathrm{Pa}(0.2 \mu$ Torr $)$ with use of a liquid-nitrogen cooled Meissner trap. The source metals are $>0.99995$ pure and are thermally evaporated from $7 \mathrm{~cm}^{3}$ crucibles at $0.1-1 \mathrm{~nm} \cdot \mathrm{s}^{-1}$ evaporation rates to produce a $0.5-0.9 \mu \mathrm{m}$ film thickness. The source-to-substrate separation of $30 \mathrm{~cm}$ enables thickness control with use of $6 \mathrm{MHz}$ gold-coated quartz crystal microbalances to produce a coating composition to within \pm 1 at.\%. The nanolaminates coatings are deposited onto $4 \mathrm{~cm} \times 8 \mathrm{~cm}$ sheets of both cleaved mica and polished Si wafers (with the native oxide intact). A $20-40 \mathrm{~nm}$ thick buffer layer of pure $\mathrm{Cu}$ is deposited initially to introduce (111) film growth. The substrate is an oxygen-free $\mathrm{Cu}$ plate that is heated with a quartz lamp for a growth temperature of $350 \pm 5^{\circ} \mathrm{C}$. The deposition process 
produces a dense columnar structure with a single habit plane of dense packed growth. The growth of (111) is typical for face-center-cubic metal alloys such as $\mathrm{Cu}-\mathrm{Ni}(\mathrm{Fe})$. The alternating layers of $\mathrm{Cu}$ and $\mathrm{Ni}(\mathrm{Fe})$ grow epitaxial with one another.

The growth or decay in amplitude $Q$ of an $\mathrm{A} / \mathrm{B}$ composition fluctuation with wavelength $\left(\lambda_{\mathrm{A} / \mathrm{B}}\right)$ for layer pairs of components $\mathrm{A}$ and $\mathrm{B}$ is accomplished again [26] using anneal treatments within a turbo-molecular pumped, quartz tube at temperatures of 320,345 , and $400{ }^{\circ} \mathrm{C}$. Samples are annealed to reduce the amplitude of the composition fluctuation from which growth or decay of the composition wave is assessed. A series of X-ray diffraction patterns are taken in the $\theta / 2 \theta$ mode, as shown in Figures 1 and 2 using a Rigaku Miniflex II diffractometer (Rigaku Americas Corp., The Woodlands, TX, USA) equipped with a graphite monochromator. A monochromatic $\mathrm{Cu} k_{\alpha}$ radiation is used to measure the amplitude and wavelength of the composition fluctuation where a $25 \mathrm{kV}-10 \mathrm{~mA}$ current was applied to the $\mathrm{Cu}$ anode. For a sample rotation $\theta$, the X-ray detector is rotated $2 \theta$. Each increment $\Delta 2 \theta$ of rotation is $0.02^{\circ}$ with a $5-10 \mathrm{~s}$ dwell time. The integrated intensity of each reflection is computed from digitized $\mathrm{X}$-ray reflectivity spectra using the diffraction peak de-convolution software provided with the Rigaku Miniflex II diffractometer. Satellite peaks $( \pm$ ) about the superlattice peak of the (111) Bragg reflection correspond with the short-range order for the composition modulation in the nanolaminate growth direction. A change in the satellite intensity $(I)$ will occur as the sample is annealed at time $(t)$ intervals that range from 20 to $1000 \mathrm{~min}$. The calculation of the composition profile is made wherein the satellite peak intensity $I^{ \pm}$is proportional with the wave amplitude. Homogenization of the structure and decay in the composition wave amplitude result for anneal treatments above the spinodal temperature, whereas growth occurs within the chemical spinodal.

\section{Method of Analysis}

A review is presented of the formulations [1-10] for spinodal decomposition that relate to use of the X-ray diffraction method for experimental measurement [21-34] of the time-temperature dependent changes in the composition wave. In particular, reference to formulations and the equations are shown which comprise the basis to compute the higher-order gradient energy coefficients, the diffusion coefficients, and the strain energy variation with nanolaminate spacing. The Background contains equations [25-28,32,33] that are now adapted for analysis of spinodal decomposition in the $\mathrm{Cu}-\mathrm{Ni}(\mathrm{Fe})$ alloy system, and as modified to determine strain energy effects in the Current Approach.

\subsection{Modified Diffusion Formulation}

The stability of a nanolaminate interface with respect to diffusion along the A/B fluctuation at time $t$ can be considered using the discrete theory in terms of a static concentration wave [32]. The composition wavelength $\lambda_{\mathrm{A} / \mathrm{B}}$ of the fluctuation between A-rich and B-rich regions, i.e., layer pair spacing for nanostructures, is equated to the wavenumber $\beta$ as $2 \pi / \lambda_{\mathrm{A} / \mathrm{B}}$. The microscopic theory of diffusion $[10,25,29]$ is used to quantify the interdiffusivity coefficient $\check{D}_{B}$ of each nanolaminate as dependent on its composition wavelength at temperature $T_{\mathrm{i}}$. The macroscopic diffusion coefficient $\check{D}$ for the alloy system corresponds with an infinite composition wavelength. The relative decay in satellite intensity $I^{ \pm}(t)$ for the composition fluctuation is normalized to the Bragg peak intensity, and this value is used to determine [22-26,33] the amplification factor $R$ as 


$$
\ln \left\{\left[I^{ \pm}(t)\right] /\left[I^{ \pm}(0)\right]\right\}=2 R \cdot t
$$

The measured intensity $I_{\mathrm{m}}$ of the Bragg peak and satellite reflections are normalized to compute corrected values $I_{\mathrm{c}}$ with respect to the $2 \theta$ peak positions. Details of the X-ray intensity formulations are provided in Appendix. The amplification factor $R$ is related to the generalized $\check{D}_{\mathrm{B}}$ through the dispersion relationship $B^{2}(\beta)$ according to the expression

$$
R=-B^{2}(\beta) \cdot \check{D}_{\mathrm{B}}
$$

The $B^{2}(h)$ expression [32] derived for face-centered-cubic [100] or [111] growth is

$$
B^{2}(h)=2\{1-\cos (2 \pi h)\} / d_{(h k l)^{2}}
$$

The interplanar spacing is $d_{(h k l)}$ and $h$ equals $d_{(h k l)} / \lambda_{\mathrm{A} / \mathrm{B}}$. The use of a curvilinear fit to the variation of $\check{D}_{\text {B with }} B^{2}(h)$ yields the value of $\check{D}$ at $B^{2}(h)=0$. That is, a nanolaminate with an infinite composition wavelength is the equivalent of a macroscopic diffusion couple. A generalized expression for $\check{D}_{\mathrm{B}}$ is presented in terms of its energetic components to account for potential nonlinear effects. The $\check{D}$ coefficient is related to $\check{D}_{\mathrm{B}}$ through $[25,26]$ the Fourier transform $F_{\mathrm{e}}(h)$ of the elastic strain energy for the distorted lattice, the second derivative $f^{\prime \prime}$ of the Helmholtz free energy (per atom volume $N_{\mathrm{v}}$ ), and the gradient-energy coefficients $K_{\mu}$ as

$$
\check{D}_{\mathrm{B}}=\check{D} \cdot\left\{1+F_{\mathrm{e}}(h) / f^{\prime \prime}+\left(2 / f^{\prime \prime}\right) \cdot \Sigma\left[K_{\mu} \cdot B^{2 \mu}(h)\right]\right\}
$$

For a face-center cubic system, $N_{\mathrm{v}}$ equals $4 / a^{3}$ for an alloy lattice parameter $a$. The order of the polynomial is $\mu$, and the diffusion coefficient $\check{D}$ is related [21] to the mobility $M$ according to the expression

$$
\check{D}=\left(M / N_{\mathrm{v}}\right) \cdot f^{\prime \prime}
$$

The $f^{\prime \prime}$ and $K_{\mu}$ terms in Equation (4) are identical with expressions [3,4,29-32] that appear in both the continuous and discrete theories of spinodal decomposition. The expression for the diffusion coefficient used in Equation (5) includes only the Helmholtz free energy term $f^{\prime \prime}$ because the presence of strain energy is not a prerequisite, but it varies with each nanolaminate sample - An original criterion of the modified diffusion equation [21] for spinodal decomposition. A direct series expansion of the $\check{D}_{\text {B }}$ with $B^{2}(h)$ relationship is now used to fit the experimental data as

$$
\check{D}_{\mathrm{B}}=\check{D} \cdot\left[1+\Sigma K_{\mu}^{\prime} \cdot B^{2 \mu}(h)\right]
$$

Accordingly, from Equations (5) and (6), the coefficients $K_{\mu}^{\prime}$ are used to compute the gradient energy coefficients $K_{\mu}$, where $K_{\mu}^{\prime}$ is now equal to $2 K_{\mu} / f^{\prime \prime}$. Equation (6) will be used in this analysis to determine the diffusion coefficient $\check{D}$, and finalize the values for both the $K_{\mu}^{\prime}$ and $K_{\mu}$ gradient energy coefficients. The $B(h)$ value is equal to $\beta$ in the long wavelength approximation. The $F_{\mathrm{e}}(h)$ quantity is equated [21] with strain energy and is, therefore, a function of $B(h)$. The strain energy term equals $2 \eta^{2} \cdot Y$, where $\eta$ is the compositionally dependent variation of strain that equals $(1 / a) \cdot(\partial a / \partial c)$, and $Y$ is the $\langle h k l>$ orientation-dependent biaxial modulus.

The diffusivity is zero at the coherent spinodal $\left(T_{\mathrm{s}}^{*}\right)$. From the linear theory using Equation (4) where only one gradient energy coefficient is considered, the following condition is found.

$$
f^{\prime \prime}+2 \eta^{2} \cdot Y+2 K \cdot B^{2}=0
$$


The gradient energy coefficient $K$ can be determined from Equation (7) with respect to the critical wavenumber where there is no growth or decay. Where the wavenumber $B$ equals zero, i.e., for a macroscopic diffusion couple where the wavelength goes to infinity, Equation (7) reduces to

$$
f^{\prime \prime}+2 \eta^{2} \cdot Y=0
$$

This relationship indicates that the $f^{\prime \prime}=0$ condition for the chemical spinodal is changed with a suppression to the condition of $f^{\prime \prime}=-2 \eta^{2} \cdot Y$ for the coherent spinodal. From an experimental estimate of the chemical spinodal temperature $T_{\mathrm{s}}$, the value of $f^{\prime \prime}$ for any temperature can then be computed [21] using Boltzmann's constant $k_{\mathrm{B}}$ as

$$
f^{\prime \prime}=N_{\mathrm{v}} \cdot k_{\mathrm{B}} \cdot\left(T-T_{\mathrm{S}}\right) /[c \cdot(1-c)]
$$

The amount that the coherent spinodal temperature is suppressed below the chemical spinodal, i.e., $T_{\mathrm{s}}-T_{\mathrm{s}}{ }^{*}$, can be determined using the Equation (8) equivalence where the temperature $T$ is now equated as $T_{\mathrm{s}}^{*}$.

\subsection{Current Approach}

In order to uniquely determine the diffusion coefficient $\check{D}$ and the unknown effect of strain energy on diffusion, the higher-order gradient-energy coefficients are now included in the expansion of the modified linear theory as seen in Equations (4) and (6). To reiterate, the suppression of the coherent spinodal described in Equations (7)-(9) will vary from sample to sample since the wavenumber-dependent Fourier transform $F_{\mathrm{e}}(h)$ of the elastic strain energy is equal to $2[\eta(h)]^{2} \cdot Y(h)$. Simulated curves are used to establish baseline behavior without inclusion of the strain energy term. This approach is taken since it has been well established for nanolaminate structures that both the strain $\eta$ and modulus $Y$ components can vary with the wavenumber, i.e., these are not constant values. In this approach, the difference between the measured data and simulated values will be used to quantify the effect of strain energy.

The value of the temperature-dependent diffusion coefficient $\check{D}$, representative of diffusion behavior for the long-wavelength equivalent, has been extrapolated previously [25-28] from the behavior of composition fluctuations with shorter wavelengths, i.e., longer wavenumbers, by fitting the variation of the interdiffusion coefficient $\check{D}_{\text {B }}$ with $B^{2}$. Without a known boundary condition, modeling of the gradient energy coefficient $K_{\mu}^{\prime}$ terms and the diffusion coefficient $\check{D}$ from the $\check{D}_{\text {B }}$ variation with $B^{2}$ behavior has introduced some uncertainty. Since all diffusion coefficients are derived from the direct measurement of the amplification factor $R$ as seen in Equation (2), the computation of the gradient energy coefficients using the microscopic theory of diffusion will now be approached by, first, using the $R$ variation with $B$ rather than the $\check{D}_{\mathrm{B}}$ variation with $B^{2}$. The value of $R$ value approaches zero for the infinite wavelength case, i.e., $B=0$, providing a boundary condition that will be used in the interpolative analysis of data to follow.

The sign conventions are reviewed to distinguish the effects of the strain energy during diffusion. Growth of the composition fluctuation when $T<T_{\mathrm{S}}$ requires that the diffusivity is negative $\left(\check{D}_{\mathrm{B}}<0\right)$ for uphill diffusion, and the amplification factor is positive $(R>0)$. Similarly, decay of the composition fluctuation when $T>T_{\mathrm{s}}$ requires that the interdiffusivity is positive $\left(\check{D}_{\mathrm{B}}>0\right)$ for downhill diffusion, and the amplification factor is negative $(R<0)$. Accordingly, from Equations (2), (4) and (5), growth of the composition wave amplitude within the spinodal occurs when $f^{\prime \prime}<0$ and $K>0$, whereas decay outside the spinodal occurs when $f^{\prime \prime}>0$ and $K<0$. 
For growth of the fluctuation, and to be consistent with the sign requirements of the model formulation reviewed in the Background, the variation of $R$ with $B$ is simulated using a new higher-order polynomial expansion,

$$
R_{\mathrm{S}}=\Sigma k_{\mu}^{\prime} \cdot B^{\mu}(h)
$$

From Equations (2) and (4), it follows that the difference $\Delta R$ between the simulated value of the amplification factor $R_{\mathrm{s}}$ and the experimental value $R_{\mathrm{e}}$, i.e., $\Delta R=R_{\mathrm{s}}-R_{\mathrm{e}}$, will be positive for all temperatures and attributable as a sole consequence of strain energy. It has been shown [21] that the strain energy accelerates diffusion outside the spinodal, but hinders it within the spinodal. So, the simulated variation of $R$ with $B$ must now envelope all experimental data as an upper bound. In addition, for decay it is already seen from Equation (10) that $\partial R / \partial B<0$ as evaluated at $B=0$, and for growth that $\partial R / \partial B>0$. These $\partial R / \partial B$ requirements guide the fitting procedure to determine the order $\mu$ of the polynomial to appropriately provide the correct $( \pm)$ sign of the first $k^{\prime} \mu$ term from Equation (10), i.e., $k^{\prime} 1<0$ for decay and $k^{\prime}{ }_{1}>0$ for growth. The $k_{\mu}^{\prime}$ terms as determined from Equation (10) are then used to compute the simulated values for interdiffusivity $\check{D}_{\text {Bs }}$ that correspond with Equation (2) as

$$
\check{D}_{\mathrm{Bs}}=-R_{\mathrm{S}} / B^{2}(h)
$$

The simulated $\check{D}_{\mathrm{Bs}}$ values are then curve fit using Equation (6) to evaluate the $K_{\mu}$ values corresponding to the fit of the $R$ variation with $B$. Since $K_{\mu}^{\prime}$ is equal to $2 K_{\mu} / f^{\prime \prime}$ from Equations (5) and (6), it follows that a condition for growth is $K_{1}^{\prime}>0$ ( since $f^{\prime \prime}<0, \check{D}<0$ and $K_{1}>0$ within the spinodal), whereas the condition for decay is $K_{1}<0$ (since $f^{\prime \prime}>0, \check{D}>0$ and $K_{1}<0$ outside the spinodal). From Equations (4)-(6), it follows that the difference $\Delta \check{D}_{\mathrm{B}}$ between the experimental value of the interdiffusion coefficient $\check{D}_{\mathrm{Be}}$ and the simulated value $\check{D}_{\mathrm{Bs}}$, i.e., $\Delta \check{D}_{\mathrm{B}}=\check{D}_{\mathrm{Be}}-\check{D}_{\mathrm{Bs}}$, will be positive for all temperatures and is a sole consequence of strain energy. Again, strain energy [21] accelerates diffusion outside the spinodal and hinders it within the spinodal. So, the simulated variation of the magnitude of the interdiffusivity $\left|\check{D}_{\mathrm{B}}\right|$ with $B$ must envelope all experimental data as a lower bound. The predetermined $K_{\mu}^{\prime}$ values that follow from evaluating Equation (6), as consistent with the Equation (10) data fit, are used to determine the one remaining, unknown diffusion parameter, i.e., $\check{D}$, at each temperature.

The determination of the $K_{\mu}^{\prime}$ values by curve fitting both the amplification factor and interdiffusivity variation with wavenumber, allows for the computation of the strain energy term consistent with previous [21] derivation. The strain energy is determined in the solution by equating the difference between Equations (4) and (11) as

$$
F_{\mathrm{e}}(h)=2 \eta(h)^{2} \cdot Y(h)=\Delta \check{D}_{\mathrm{B}} \cdot\left[f^{\prime \prime}(h) / \check{D}\right]
$$

A plot of the strain energy term $2 \eta^{2} \cdot Y$ can then be made to evaluate its variation with composition wavelength $\lambda$.

\section{Results and Discussion}

The growth of structures with longer wavelengths, i.e., smaller wavenumbers, occurs in the $\alpha$-phase for temperatures within the spinodal to produce phase separated regions of $\alpha^{\prime}$ and $\alpha^{\prime \prime}$ with dissimilar lattice spacing. The consequence for a bulk $\alpha$-phase alloy will be a tendency to split the Bragg X-ray reflection, decrease its intensity, and broaden its shoulder as is consistent with the growth of long 
wavelength components. However, for nanolaminate structures, there is an initial composition wavelength along a specific crystallographic direction. With annealing, the $\mathrm{Cu} / \mathrm{Ni}(\mathrm{Fe})$ superlattice $\mathrm{Bragg}$ peak may first narrow in width due to the effects of nanocrystalline grain growth, and split due to phase separation where a $2 \theta_{\mathrm{Cu}}$ peak may appear, as seen in Figures 1 and 2 for 2.1 and $8.9 \mathrm{~nm}$ wavelength samples annealed at $400{ }^{\circ} \mathrm{C}$ for $40 \mathrm{~min}$. The satellite peaks that correspond with the composition fluctuation are indicated in the subscript of the $2 \theta_{\mathrm{i}}$ peak position in Figures 1 and 2.

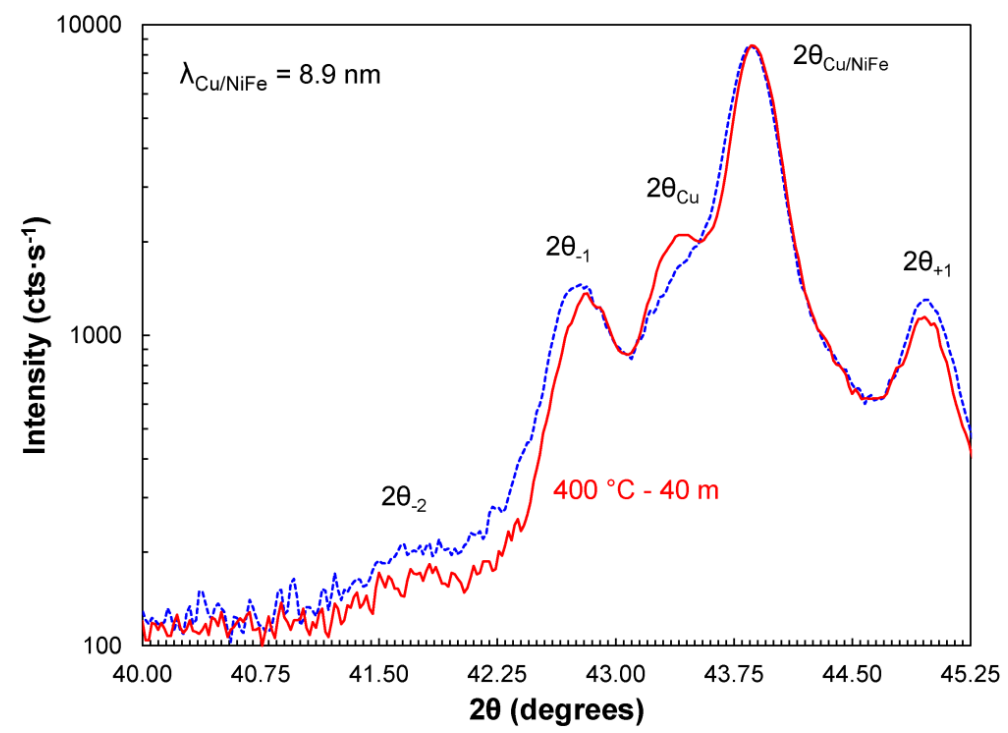

Figure 1. The intensity $I^{ \pm}$of the Bragg and satellite peak variation with $2 \theta$ position for the $\mathrm{Cu} / \mathrm{Ni}(\mathrm{Fe})$ nanolaminate coating with a $8.9 \mathrm{~nm}$ wavelength $\lambda$ before (dashed line) and after (solid line) a $40 \mathrm{~min}$ at $400{ }^{\circ} \mathrm{C}$ anneal treatment.

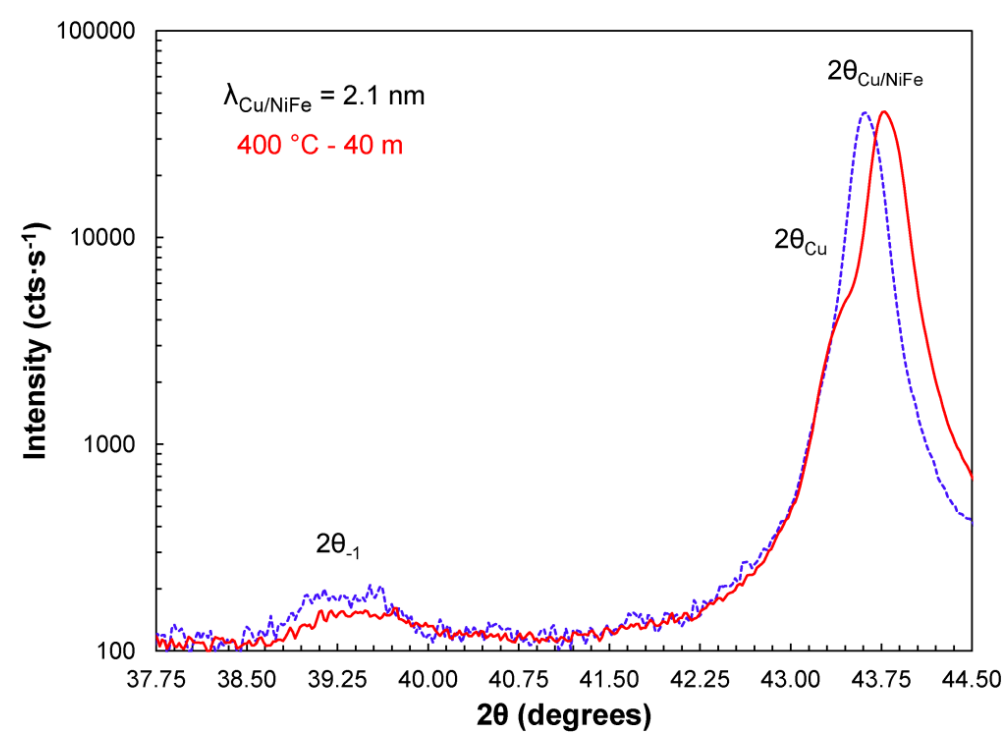

Figure 2. The intensity $I^{ \pm}$of the Bragg and satellite peak variation with $2 \theta$ position for the $\mathrm{Cu} / \mathrm{Ni}(\mathrm{Fe})$ nanolaminate coating with a $2.1 \mathrm{~nm}$ wavelength $\lambda$ before (dashed line) and after (solid line) a 40 min at $400{ }^{\circ} \mathrm{C}$ anneal treatment.

The relaxation of lattice strain (when present) can shift the Bragg peak position as seen for the $2.1 \mathrm{~nm}$ nanolaminate sample of Figure 2. In addition, enhanced diffusivity for an artificial composition wave 
during the early stages of annealing [24-26] causes an initial, nonlinear decay of satellite peak intensity with time.

The amplification factor $R$ variation for the nanolaminates (at each anneal temperature) is computed from the integrated peak intensities of the diffraction spectra as shown in Figures 1 and 2 using Equation (1). The $R$-values are plotted in Figure 3 as a function of the dispersion relation $B$ which is computed using Equation (3) for a (111) growth of the $\mathrm{Cu} / \mathrm{Ni}(\mathrm{Fe})$ structure. Decay of the composition fluctuation occurs above $345^{\circ} \mathrm{C}$ and growth below $320^{\circ} \mathrm{C}$, providing an interpolated estimate for the chemical spinodal of $335^{\circ} \mathrm{C}$. There is insufficient temperature-dependent data in this study to detail the effects of composition wavelength on the depression of the spinodal due to coherency effects. However, a critical wavelength $\lambda_{\mathrm{c}}$ of $1.26 \mathrm{~nm}$ is found for growth at $320^{\circ} \mathrm{C}$, i.e., the coherent spinodal temperature $T_{\mathrm{s}}{ }^{*}$ is $320{ }^{\circ} \mathrm{C}$ for a $1.26 \mathrm{~nm}$ composition wavelength. This corresponds with the $B_{\mathrm{c}}$ value of $4.78 \mathrm{~nm}^{-1}$ where $R_{\lambda}$ equals zero as determined from the Equation (10) fitting of the $R$-values in Figure 3 . This $\lambda_{\mathrm{c}}$-value corresponds with a composition wavelength for maximum growth $\lambda_{\mathrm{m}}$ of $1.78 \mathrm{~nm}$ at $320^{\circ} \mathrm{C}$, where [21] $\lambda_{m}$ equals $\lambda_{c} \cdot \sqrt{2}$.

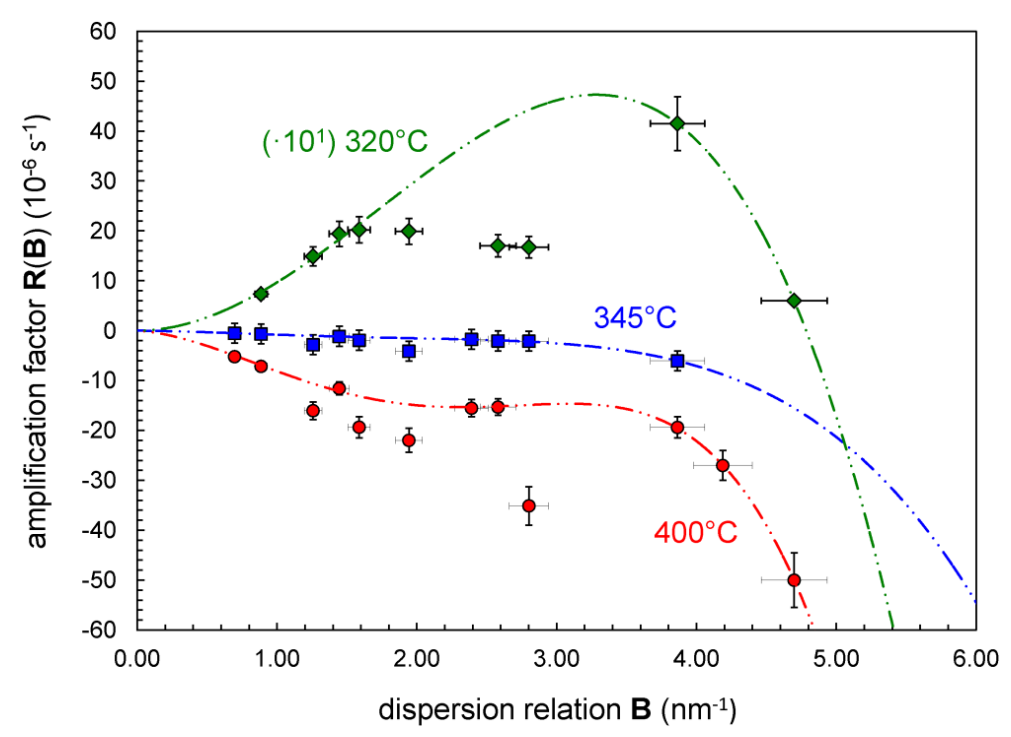

Figure 3. The amplification factor $R\left(10^{6} \mathrm{~s}^{-1}\right)$ variation with the dispersion-relation wavenumber $B\left(\mathrm{~nm}^{-1}\right)$ as computed from the decay or growth of the first-order satellite intensity $I^{-}$at peak position of $2 \theta_{-1}$ for $\mathrm{Cu} / \mathrm{Ni}(\mathrm{Fe})$ nanolaminate samples annealed at $320^{\circ} \mathrm{C}$ (diamond) as scaled by a factor of 10 , at $345^{\circ} \mathrm{C}$ (squares), and $400{ }^{\circ} \mathrm{C}$ (circles).

The $f^{\prime \prime}$ values can next be computed, as listed in Table 1, using Equation (9) where $T_{\mathrm{s}}$ equals $335^{\circ} \mathrm{C}$ for $\mathrm{Cu}_{0.53} / \mathrm{Ni}_{0.40} \mathrm{Fe}_{0.07}$ using a $N_{\mathrm{v}}$ value of 86.1 atom $\cdot \mathrm{nm}^{-3}$ as computed for 4 atoms in a unit cell volume with a $0.3595 \mathrm{~nm}$ lattice parameter. A positive $f^{\prime \prime}$ value is consistent with the heat of mixing wherein unlike atoms cluster as opposed to having the tendency for long-range ordering for systems with a negative heat of mixing. The baseline $R$ behavior for growth or decay is modeled as a function of $B$ using a fourth-order polynomial expansion of Equation (10). This is the minimum order of expansion required to meet the sign criteria for the modeled $k_{\mu}^{\prime}$ values that is described in the Current Approach Section 4.2. 
Table 1. Diffusion and gradient energy coefficients for $\mathrm{Cu} / \mathrm{Ni}(\mathrm{Fe})$ nanolaminates.

\begin{tabular}{ccccc}
\hline Diffusion and Gradient Energy Coefficients & & $\mathbf{C u} / \mathbf{N i}(\mathbf{F e})$ & & $\mathbf{C u} / \mathbf{N i}$ \\
\hline$T\left({ }^{\circ} \mathrm{C}\right)$ & 320 & 345 & 400 & 400 \\
$\check{D}\left(10^{-6} \mathrm{~nm}^{2} \cdot \mathrm{s}^{-1}\right)$ & -1.065 & 1.208 & 11.27 & 4.846 \\
$M\left(10^{+16} \mathrm{~nm}^{2} \cdot \mathrm{J}^{-1} \cdot \mathrm{s}^{-1}\right)$ & 0.1285 & 0.2187 & 0.3139 & 0.1350 \\
$f^{\prime \prime}\left(10^{-19} \mathrm{~J} \cdot \mathrm{nm}^{-3}\right)$ & -0.7134 & 0.4756 & 3.091 & 3.078 \\
$K_{1}\left(10^{-20} \mathrm{~J} \cdot \mathrm{nm}^{-1}\right)$ & 0.3496 & -0.9311 & -4.494 & -2.650 \\
$K_{2}\left(10^{-21} \mathrm{~J} \cdot \mathrm{nm}^{3}\right.$ & -0.2735 & 1.890 & 5.752 & 2.582 \\
$K_{3}\left(10^{-22} \mathrm{~J} \cdot \mathrm{nm}^{3}\right)$ & 0.1496 & -1.702 & -3.991 & -0.9814 \\
$K_{4}\left(10^{-23} \mathrm{~J} \cdot \mathrm{nm}^{5}\right)$ & -0.0295 & 0.5397 & 0.6091 & 0.1309 \\
\hline
\end{tabular}

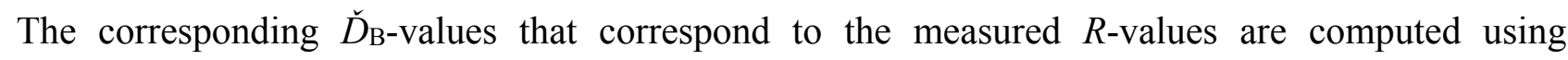
Equation (2) and are plotted in Figure 4. The baseline $\check{D}_{\mathrm{B}}$ behavior for growth or decay is computed as a function of $B^{2}$ using the $k_{\mu}^{\prime}$ coefficients. The $K_{\mu}^{\prime}$ coefficients are determined by using a fourth-order expansion of Equation (6) to fit the baseline $\check{D}_{\mathrm{B}}$-values. This is the minimum order needed to meet the sign criteria for the modeled $K_{\mu}^{\prime}$ values as described in the Current Approach Section 4.2. From this data fit that prescribes conversion of $k_{\mu}^{\prime}$ into $K_{\mu}^{\prime}$ coefficients, the remaining unknown, i.e., the intercept value for $\check{D}$, is uniquely determined. The corresponding $K_{\mu}$ values of Equation (4) are listed in Table 1, where it has been noted previously that $K_{\mu}^{\prime}$ equals $2 K_{\mu} / f^{\prime \prime}$. The mobility $M$ values at each temperature are listed in Table 1 as computed using Equation (5). In general, the mobility $M$ will decrease with a decreasing value of the $\check{D} / f^{\prime \prime}$ ratio.

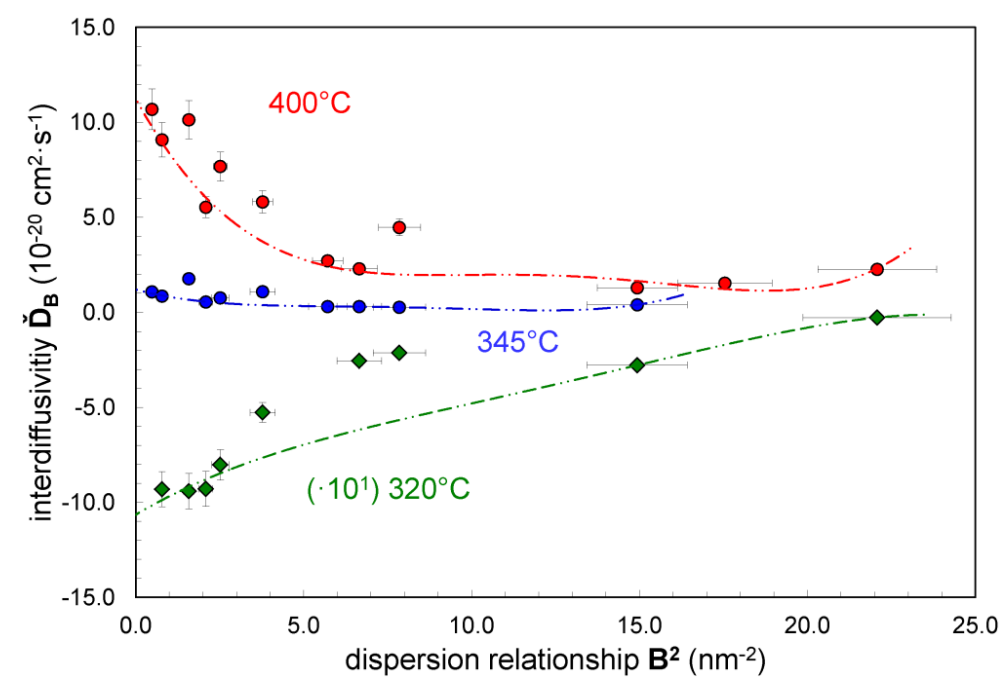

Figure 4. The interdiffusivity $\check{D}_{\mathrm{B}}\left(\mathrm{cm}^{2} \cdot \mathrm{s}^{-1}\right)$ variation with the square of the dispersion relation wavenumber $B^{2}\left(\mathrm{~nm}^{-2}\right)$ as computed from the amplification factor $R$ for $\mathrm{Cu} / \mathrm{Ni}(\mathrm{Fe})$ nanolaminate samples annealed at $320^{\circ} \mathrm{C}$ (diamond) as scaled by a factor of 10 , at $345^{\circ} \mathrm{C}$ (squares), and $400{ }^{\circ} \mathrm{C}$ (circles).

The strain energy component, that primarily constitutes [21] the Fourier energy $F_{\mathrm{e}}$, is computed using Equation (12). The energy variation with composition wavelength is plotted in Figure 5. It is clear that a strain energy contribution provides a significant effect in some nanolaminates by enhancing the decay of the composition fluctuation outside the spinodal and hindering its decay within the spinodal as seen 
in the results of Figures 3 and 4 . The peak (strain) energy computed for the $\mathrm{Cu} / \mathrm{Ni}(\mathrm{Fe})$ nanolaminates has a value of $0.94 \times 10^{8} \mathrm{~J} \cdot \mathrm{m}^{-3}$. The magnitude for this energetic component as seen in Figure 5 is comparable to the values of the Helmholtz free-energy term $f^{\prime \prime}$ that are listed in Table 1. In comparison, a smaller $2 \eta^{2} \cdot Y$ value of $0.43 \times 10^{8} \mathrm{~J} \cdot \mathrm{m}^{-3}$ can be computed for $\mathrm{Cu} / \mathrm{Ni}(\mathrm{Fe})$ using a rule-of-mixtures formulation with a biaxial modulus $Y$ of $200 \mathrm{GPa}$ and a $2 \eta^{2}$ strain term of $2.14 \times 10^{-4}$ assuming a $1.03 \%$ interface misfit strain.

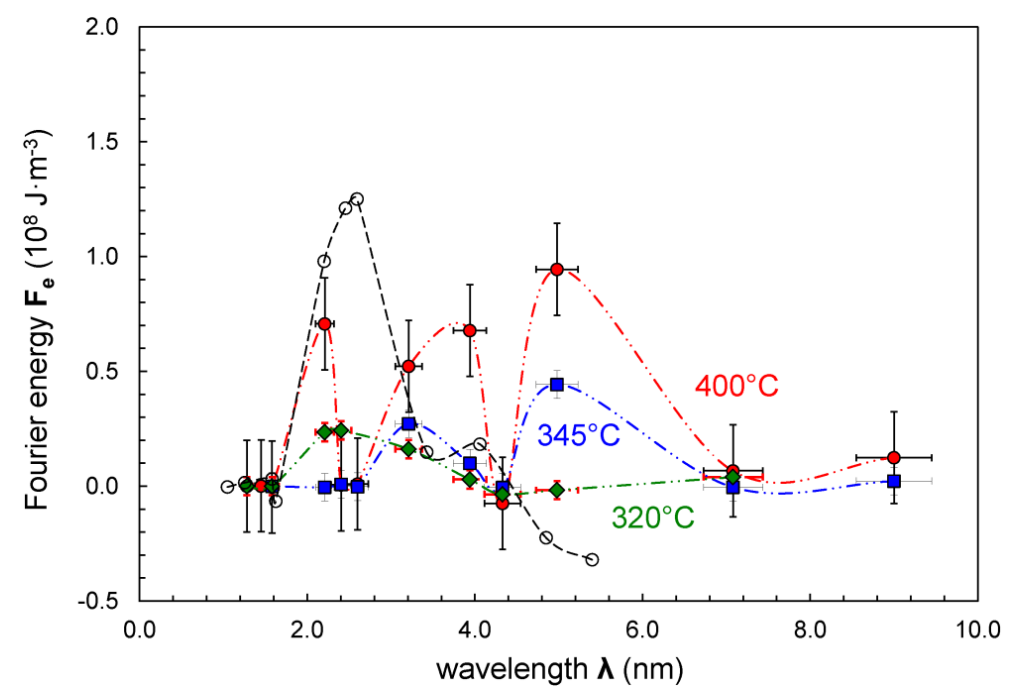

Figure 5. The Fourier energy $F_{\mathrm{e}}\left(10^{8} \mathrm{~J} \cdot \mathrm{m}^{-3}\right)$ variation with composition wavelength $\lambda(\mathrm{nm})$ as computed from the difference between experimental values and the modeled fit of the amplification factor $R$ variation with the dispersion relation wavenumber $B\left(\mathrm{~nm}^{-1}\right)$ for nanolaminate samples of: $\mathrm{Cu} / \mathrm{Ni}(\mathrm{Fe})$ annealed at $320^{\circ} \mathrm{C}$ (diamond), $345{ }^{\circ} \mathrm{C}$ (squares), and $400{ }^{\circ} \mathrm{C}$ (solid circles); as well as $\mathrm{Cu} / \mathrm{Ni}$ annealed at $400{ }^{\circ} \mathrm{C}$ (open circles).

\section{Analysis and Discussion}

Single and multiple peaks in the interdiffusivity variation with wavenumber are reported [24,25,35] for the diffusion results of the $\mathrm{Cu} / \mathrm{Ni}, \mathrm{Cu} / \mathrm{Pd}, \mathrm{Au} / \mathrm{Cu}$, and $\mathrm{Ag} / \mathrm{Pd}$ nanolaminate systems. The multiple fluctuations observed in both the amplification of Figure 3 and the interdiffusivity of Figure 4 are a potential consequence of: a shift towards longer wavelengths that can occur with aging time; and that strain energy will still enhance (outside the spinodal) or hinder (within the spinodal) diffusion at the shorter wavelengths. As introduced in Section 4.2, some data points in Figures 3 and 4 will vary from the simulated curves that are used to fit and establish the baseline behavior without the strain energy term. Consequently a variation in the Fourier energy $F_{\mathrm{e}}$, is an anticipated result, i.e., the strain energy dependence on composition wavelength as plotted in Figure 5. It is seen here that the magnitude of $F_{\mathrm{e}}$ measured for the $\mathrm{Cu} / \mathrm{Ni}(\mathrm{Fe})$ nanolaminates decreases somewhat with reduced annealing temperature. In addition, computations of $F_{\mathrm{e}}$ for the $\mathrm{Cu}-\mathrm{Ni}$ nanolaminate system from interdiffusion data reported at $400{ }^{\circ} \mathrm{C}[25]$ are plotted (with open circles) in Figure 5 using the present model approach for determining the gradient energy and diffusion coefficients as listed in Table 1 . The $\mathrm{Cu} / \mathrm{Ni}$ data shows a bimodal distribution with a peak value of $1.25 \cdot 10^{8} \mathrm{~J} \cdot \mathrm{m}^{-3}$ at a composition wavelength of $2.6 \mathrm{~nm}$. The strain energy effects will be lost for very-long composition wavelengths where strained-layer superlattice 
growth is difficult due to the introduction of threading dislocations, and for very short $(<1 \mathrm{~nm})$ wavelengths where there is elastic homogenization from interface mixing. Therefore, strain energy will vary with the composition wavelength from $\sim 1$ to $9 \mathrm{~nm}$. Periodic or irregular variations in strain energy may result, as well, for nanolaminate structures that do not have a commensurate structure. A variety of X-ray techniques and analysis methods [36-41] are available to assess strain distributions through surface regions and strained-layer superlattices. However, a detailed assessment for profiling of elastic strains and the variation through the interface is beyond the scope of this present analysis.

The Arrhenius plot of $\check{D}$ versus $T^{-1}$ in Figure 6 is used to compare the $|\check{D}|$ values of the $\mathrm{Cu}_{0.53} / \mathrm{Ni}_{0.40} \mathrm{Fe}_{0.07}$ nanolaminates to the bulk diffusion measurements of $\mathrm{Cu}_{0.877} \mathrm{Ni}_{0.107} \mathrm{Fe}_{0.016}$ [42] and $\mathrm{Cu}_{0.5} \mathrm{Ni}_{(0.5-x)} \mathrm{Fe}_{x}$ where $0.02<x<0.08$ [43,44] alloys. An activation energy $Q$ of $51 \pm 2 \mathrm{kcal} / \mathrm{mol}$ $(215 \pm 6 \mathrm{~kJ} / \mathrm{mol} ; 1.34 \pm 0.06 \mathrm{eV} /$ atom) is determined from the slope of a linear fit to the $\mathrm{Cu}_{0.877} \mathrm{Ni}_{0.107} \mathrm{Fe}_{0.016}$ data. This linear fit provides an upper bound to the bulk measurements, and is consistent with the new $|\check{D}|$ values for the nanolaminates as computed from Equations (6), (10) and (11). It has been postulated [21] that an asymptotic discontinuity is possible at the spinodal temperature, where the magnitude of the diffusion coefficient $|\check{D}|$ vanishes since the composition fluctuation neither grows nor decays at $T_{\mathrm{c}}$. Curvilinear (dashed) lines are constructed in Figure 6 where the diffusion values drop below the upper bound linear curve. A local depression in $|\check{D}|$ is seen near a $10^{3} / T$ value of $\sim 1.6$ which corresponds with a spinodal temperature of $\sim 350{ }^{\circ} \mathrm{C}$. This value for the spinodal temperature is close to the $335^{\circ} \mathrm{C}$ assumed in the computation of $f^{\prime \prime}$ using Equation (9), which is similar to the $333{ }^{\circ} \mathrm{C}$ result from a thermodynamic evaluation [45] of the $\mathrm{Cu}-\mathrm{Ni}$ alloy system using the CALPHAD approach. In an assessment of the collective interdiffusion in both Fe- and Si-based nanolaminate structures [46], it has been proposed that a collective atomic jumping mechanism that governs interdiffusion involves at least $8-15$ atoms.

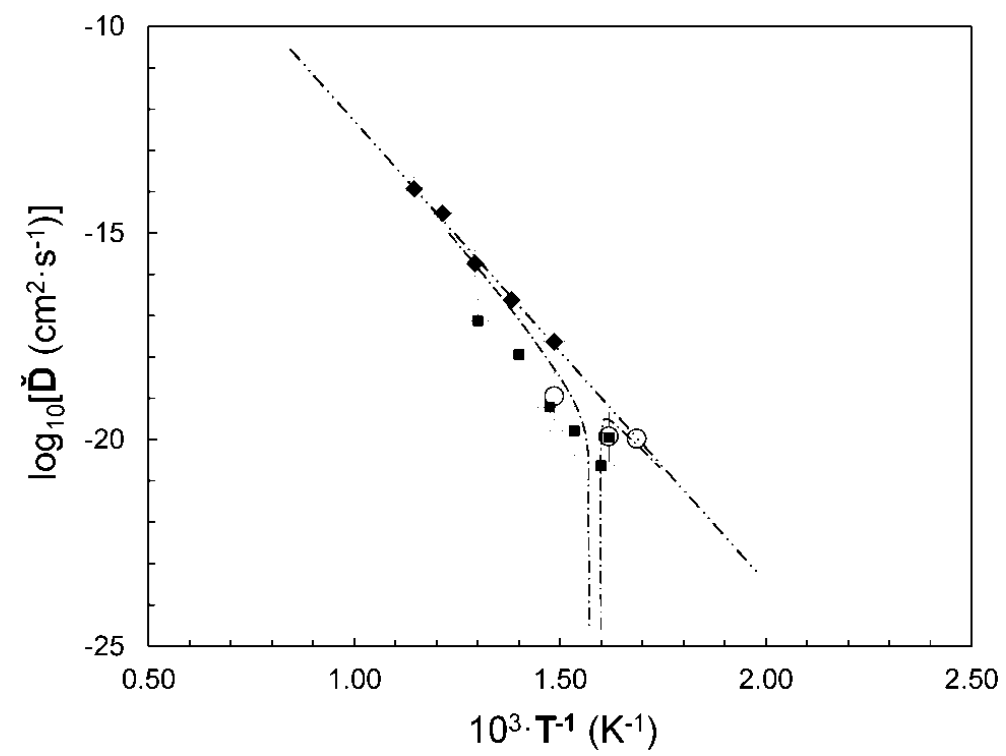

Figure 6. The Arrhenius-type relationship is plotted for the $\log _{10}$-scale variation of diffusion $\check{D}\left(\mathrm{~cm}^{2} \cdot \mathrm{s}^{-1}\right)$ with inverse temperature $10^{3} \cdot T^{-1}\left(\mathrm{~K}^{-1}\right)$ for the $\mathrm{Cu} / \mathrm{Ni}(\mathrm{Fe})$ nanolaminates (open circles) along with experimental data for $\mathrm{Cu}_{0.877} \mathrm{Ni}_{0.107} \mathrm{Fe}_{0.016}$ (solid diamonds) [33] and

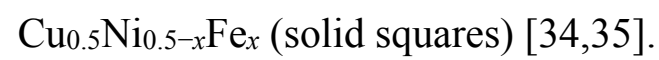




\section{Conclusions}

The static concentration wave approach to diffusion is used to model the diffusivity behavior for the spinodal decomposition of $\mathrm{Cu} / \mathrm{Ni}(\mathrm{Fe})$ nanolaminates. In a comparison of results for aging at 320,345 and $400{ }^{\circ} \mathrm{C}$, growth of the composition fluctuation is seen within the spinodal at temperatures below $\sim 335^{\circ} \mathrm{C}$. A potential asymptote in an Arrhenius plot of the diffusion coefficients $\check{D}$ (as modeled for the long-wavelength approximation) is consistent with drop off in diffusivity that would occur near the chemical spinodal temperature of $\sim 335^{\circ} \mathrm{C}$. The gradient energy coefficient is expanded as a fourth-order polynomial function of the wavenumber for the composition fluctuation. A fitting procedure that starts with the variation of the amplification factor takes advantage of the boundary condition where growth or decay vanishes at infinite wavelength. The sign of the gradient-energy coefficient $K_{\mu}$ alternates with increasing $\mu$-order, starting with a 1st-order coefficient that has: a negative value outside the chemical spinodal, and; a positive value within the spinodal in accordance with the Cahn-Hilliard theory for spinodal decomposition. The magnitude of the gradient-energy coefficients decrease with: the increasing $\mu$-order of the interaction and; decreasing temperature. A maximum magnitude of $4.49 \times 10^{-20} \mathrm{~J} \cdot \mathrm{nm}^{-1}$ for $K_{1}$ is found at $400{ }^{\circ} \mathrm{C}$ and a minimum magnitude of $0.03 \times 10^{-23} \mathrm{~J} \cdot \mathrm{nm}^{5}$ for $K_{4}$. Fluctuation in the strain energy $2 \eta^{2} \cdot Y$ term is seen for nanolaminate structures with composition wavelengths $\lambda$ less than $7 \mathrm{~nm}$, where a peak value of $0.94 \times 10^{8} \mathrm{~J} \cdot \mathrm{m}^{-3}$ is found from analysis of diffusion data at an aging temperature of $400{ }^{\circ} \mathrm{C}$. A decrease in the strain energy for $\mathrm{Cu} / \mathrm{Ni}(\mathrm{Fe})$ is found outside the $2-5 \mathrm{~nm}$ range of composition wavelengths - As is consistent with a typical loss in strained-layer superlattice growth for metallic nanolaminates as often attributed to intermixing at interfaces and the introduction of threading dislocations.

\section{Acknowledgments}

This work was supported by a Texas Tech University faculty development leave of absence.

\section{Conflicts of Interest}

The author declares no conflict of interest.

\section{Appendix}

Integrated intensity values $I_{\mathrm{c}}$ are corrected from measured $I_{\mathrm{m}}$ values through consideration [24,25] of effects as composition-averaged scattering, polarization, mass absorption and temperature according to the expression.

$$
I_{\mathrm{c}}=\left(I_{\mathrm{m}}\right) /\left(F^{2} \cdot L \cdot P \cdot T_{x} \cdot D_{\mathrm{w}}\right)
$$

The measured intensities are normalized with respect to the following factors: $F$-For the composition-averaged atomic scatterings $F_{\mathrm{i}} ; L \cdot P$ - The Lorentz-Polarization effect as corrected for a graphite monochromator that, in this case, is found at a Bragg angle $2 \alpha$ of $25^{\circ} ; T_{x}$-For the product of the mass absorption coefficient $\mu / \rho$ with density $\rho$ and specimen film thickness $x$; and $D_{\mathrm{w}}$-The Debye-Waller temperature effect on lattice vibration. The $F^{2} \cdot L \cdot P$ product dominates the intensity normalization. For the pseudobinary $\mathrm{Cu}-\mathrm{Ni}(\mathrm{Fe})$ alloy that consists of $\mathrm{Cu}$ and $\mathrm{Ni}(\mathrm{Fe})$ layers, the constituent terms of Equation (A.1) are written as. 


$$
\begin{gathered}
F=c \mathrm{Cu} \cdot F_{\mathrm{Cu}}+c \mathrm{NiFe} \cdot F_{\mathrm{NiFe}}=c \mathrm{Cu} \cdot F_{\mathrm{Cu}}+(1-c \mathrm{Cu}) \cdot F_{\mathrm{NiFe}} \\
F_{\mathrm{Cu}}=18.9-3.3 \cdot[\sin (\theta) / \lambda \mathrm{X} \text {-ray }-2] \\
F_{\mathrm{Ni}}=20.7-3.5 \cdot[\sin (\theta) / \lambda \mathrm{X} \text {-ray }-2] \\
F_{\mathrm{Fe}}=21.6-3.7 \cdot[\sin (\theta) / \lambda \mathrm{X} \text {-ray }-2] \\
F_{\mathrm{NiFe}}=\mathrm{c} \cdot F_{\mathrm{Fe}}+(1-\mathrm{c}) \cdot F_{\mathrm{Ni}} \\
c=c \mathrm{Fe} /(c \mathrm{Fe}+c \mathrm{Ni}) \\
T_{x}=1-\mathrm{e}^{[-2 \mu \cdot x / \sin (\theta)]} \\
D_{\mathrm{w}}=\mathrm{e}^{[-2 \underline{\mathrm{B}} \cdot H]} \\
H=[\sin (\theta) / \lambda \mathrm{X} \text {-ray }]^{2} \\
\underline{\mathrm{B}}=(2 \pi \cdot \hat{u})^{2}
\end{gathered}
$$

In Equations (A.2.9)-(A.2.11), the magnitude of displacement is $\hat{u}$ for the scattering vector. The time dependent amplitude $Q(t)$ for the $\mathrm{A} / \mathrm{B}$ composition modulation of wavelength $\lambda_{\mathbf{A} / \mathbf{B}}$ with respect to the corrected time-dependent intensity $I(t)$ variation is expressed [24] as

$$
\left[I^{ \pm}(t) / I^{\mathrm{B}}(t)\right]_{\mathrm{c}}=\left\{1 / 2 Q(t) \cdot\left[\left(\lambda_{\mathrm{A} / \mathrm{B}} \cdot \varepsilon_{0} \cdot s^{ \pm}\right) \pm(-\xi)\right]\right\}^{2}
$$

The terms in Equation (A.3) are defined as

$$
\begin{gathered}
s^{ \pm}=\left[2 \cdot \sin \left(\theta^{\mathrm{B}}\right) / \lambda_{\mathrm{X} \text {-ray }}\right] \pm\left(1 / \lambda_{\mathrm{A} / \mathrm{B}}\right) \\
s^{ \pm}=2 \cdot \sin \left(\theta^{ \pm}\right) / \lambda \mathrm{X} \text {-ray } \\
\varepsilon_{\mathrm{o}}=\left[\left(\xi^{+} \cdot w^{-}\right)+\left(\xi^{-} \cdot w^{+}\right)\right] /\left\{\lambda_{\mathrm{A} / \mathrm{B}} \cdot\left[\left(s^{+} \cdot w^{-}\right)-\left(s^{-} \cdot w^{+}\right)\right]\right\} \\
\xi=\Delta F / F \\
\Delta F=F_{\mathrm{A}}-F_{\mathrm{B}}=F_{\mathrm{Cu}}-F_{\mathrm{NiFe}} \\
w^{ \pm}=\left(I^{ \pm} / I^{\mathrm{B}}\right)^{1 / 2}
\end{gathered}
$$

The $\varepsilon_{o}$ parameter is the amplitude of the lattice modulation per unit composition amplitude. $Q(t)$ can be expressed by combining Equations (A.3) and (A.4.1-5). The assumption can be made [4] that the variation in the absolute value of the scattering factor $\left(F_{\mathrm{i}}\right)$ between the Bragg and first-order satellite reflections is negligible, i.e., $\xi \approx \xi^{+} \approx \xi^{-}$. The expression for $Q(t)$ then reduces to.

$$
Q(t)=\left[\left(s^{+} \cdot w^{-}\right)-\left(s^{-} \cdot w^{+}\right)\right] /\left(s^{\mathrm{B}} \cdot \xi\right)
$$




\section{References}

1. Cahn, J.W.; Hilliard, J.E. Free energy of a nonuniform system. I. Interfacial free energy. J. Chem. Phys. 1958, 28, 258-267.

2. Hillert, M.A. A solid-solution model for inhomogeneous systems. Acta Metall. 1961, 9, 525-535.

3. Cahn, J.W. On spinodal decomposition. Acta Metall. 1961, 9, 795-801.

4. Cahn, J.W. On spinodal decomposition in cubic crystals. Acta Metall. 1962, 10, 179-183.

5. Cahn, J.W. Coherent fluctuations and nucleation in isotropic solids. Acta Metall. 1962, 10, 907-913.

6. Khachaturyan, A.G. Application of the method of two-time Green functions to the problem of ordering alloys. Fiz. Met. Metalloved. 1962, 13, 493-501.

7. Khachaturyan, A.G. Application of the Green's function method to the thermodynamics of interstitial solutions. Sov. Phys. Solid State 1963, 5, 9-12.

8. Cahn, J.W. The Effects of an Applied Stress on Spinodal Decomposition. Report No. 64-RL-3561M. General Electric Research Laboratory, Schenectady, NY, USA, 1964.

9. Khachaturyan, A.G. On the theory of modulated structures in binary solid solutions. Sov. Phys. Crystallogr. 1965, 10, 248-255.

10. Khachaturyan, A.G. Microscopic theory of diffusion in crystalline solid solutions and the time evolution of the diffuse scattering of X-rays and thermal neutrons. Sov. Phys. Solid State 1968, 9 , 2040-2046.

11. Fraerman, A.A.; Mel'nikov, A.S.; Nefedov, I.M.; Shereshevskii, I.A.; Shpiro, A.V. Nonlinear relaxation dynamics in decomposing alloys: One-dimensional Cahn-Hilliard model. Phys. Rev. B 1997, 55, 6316-6323.

12. Pyataev, A.V.; Manapov, R.A. Mössbauer study of the process of the room-temperature aging of the alloy $\mathrm{Cu}_{79} \mathrm{Ni}_{14} \mathrm{Fe}_{7}$. Phys. Met. Metall. 2011, 111, 38-44.

13. Hernandez-Santiago, F.; Espinoza-Ramirez, I.; Lopez-Hirata, V.M.; Saucedo-Muňoz, M.L.; Diaz-Barriga-Arceo, L.; Dorantes-Rosales, H.J. Phase decomposition in isothermally aged MA $\mathrm{Cu}-\mathrm{Ni}-\mathrm{Fe}$ and $\mathrm{Cu}-\mathrm{Ni}-\mathrm{Cr}$ alloys. Adv. Mater. Res. 2007, 15, 678-683.

14. Dahlgren, S.D. Correlation of yield strength with internal coherency strains for age-hardned Cu-Ni-Fe alloys. Metal. Trans. A 1977, 8, 347-351.

15. $\mathrm{Xu}, \mathrm{Z}$. Strengthening mechanism of modulated structure initiated by spinodal decomposition, Acta Metal. Sin. 2011, 47, 1-6.

16. Jirapure, S.C.; Borade, A.B.; Kongre, U.V. Spinodal Decomposition-An Effective Method of Strengthening. Int. J. Adv. Technol. Eng. Sci. 2015, 3, 209-219.

17. Jin, S.; Chen, L.H.; Tiefel, T.H.; Eibschutz, M. Modulation-induced giant magnetoresistance in a spinodally decomposed Cu-Ni-Fe alloy. J. Appl. Phys. 1994, 75, 6915-6917.

18. Zhou, Q.; Jiang, J.; Zhong, Q.; Wang, Y.; Li, K.; Lu, H. Preparation of Cu-Ni-Fe alloy coatings and its evaluation on corrosion behavior in 3.5\% NaCl. J. Alloy. Comp. 2013, 563, 171-175.

19. Esmaili, S.; Bahrololoom, M.E.; Zamani, C. Giant Magnetoresistance and Super-Paramagnetism in Electrodeposited NiFe/Cu Multilayers. Surf. Eng. Appl. Electrochem. 2011, 47, 323-327.

20. Bradley, A.J.; Cox, W.F.; Goldschmidt, H.J. An X-ray study of the iron-copper-nickel equilibrium diagram at various temperatures. J. Inst. Met. 1941, 67, 189-201. 
21. Hilliard, J.E. Phase Transformations; Aaronson, H.I., Ed.; ASM: Metals Park, OH, USA, 1970; pp. 497-559.

22. Dumond, J.; Youtz, J.P. An X-ray method of determining rates of diffusion in the solid state. J. Applied Phys. 1940, 11, 357-365.

23. Cook, H.E.; Hilliard, J.E. Effect of gradient energy on diffusion in gold-silver alloys. J. Appl.Phys. 1969, 40, 2191-2198.

24. Paulson, W.M.; Hilliard, J.E. Interdiffusion in composition-modulated gold-copper thin films. J. Applied Phys. 1977, 48, 2117-2123.

25. Tsakalakos, T. Composition-modulated films: New materials for studying stability and critical phenomena in solid solutions. Thin Solid Films 1981, 86, 79-90.

26. Jankowski, A.F.; Tsakalakos, T. Phase stability by the artificial concentration wave method. Metall. Trans. A 1989, 20, 357-362.

27. Jankowski, A.F.; Saw, C.K. Diffusion in Ni/CrMo composition modulated films. Scr. Mater. 2004, 51, 119-124.

28. Jankowski, A.F. Diffusion mechanisms in nanocrystalline and nanolaminate Au-Cu. Defect Diffus . Forum 2007, 266, 13-28.

29. Cook, H.E.; de Fontaine, D.; Hilliard, J.E. A model for diffusion on cubic lattices and its applications to the early stages of ordering. Acta Metall. 1969, 17, 765-773.

30. Cook, H.E.; De Fontaine, D. On the elastic free energy of solid solutions-I. Microscopic theory. Acta Metall. 1969, 17, 915-924.

31. Khachaturyan, A.G.; Pokrovskii, B.I. Concentration wave approach in structural and thermodynamic characterization of ceramic crystals. Prog. Mater. Sci. 1985, 29, 1-138.

32. Khachaturyan, A.G. Theory of Structural Transformations in Solids; John Wiley and Sons: New York, NY, USA, 1983.

33. Guinier, A. X-ray Diffraction in Crystals, Imperfect Crystals and Amorphous Bodies; W.H. Freeman: San Francisco, CA, USA, 1963.

34. Cullity, B.D. Elements of X-ray Diffraction; Addison Wesley: Reading, UK, 1978.

35. Philofsky, E.M.; Hilliard, J.E. Effect of coherency strains on diffusion in copper-palladium alloys. J. Appl. Phys. 1969, 40, 2198-2205.

36. Speriosu, V.S.; Vreeland, T., Jr. X-ray rocking curve analysis of superlattices. J. Appl. Phys. 1984, 56, 1591-1600.

37. Olego, D.J.; Shahzad, K.; Petruzzello, J.; Cammack, D. Depth profiling of elastic strains in lattice-mismatched semiconductor heterostructures and strained-layer superlattices. Phys. Rev. B 1987, 36, 7674-7677.

38. Mitura, Z.; Mikołajczak, P. Computer simulation of X-ray spectra of metallic superlattices. J. Phys. F Met. Phys. 1988, 18, 183-195.

39. Jankowski, A.F. The strain wave approach to modulus enhancement and stability of metallic multilayers. J. Phys. Chem. Solids 1989, 50, 641-649.

40. Tsakalakos, T.; Khachaturyan, A.G. Interfacial displacements in multilayers with similar lattices. Mater. Sci. Eng. B 1990, 6, 123-127.

41. Chaudhuri, J.; Gondhalekar, V.; Jankowski, A.F. X-ray diffraction analysis of Au/Ni multilayers. J. Appl. Phys. 1992, 71, 3816-3820. 
42. Gust, W.; Wachtel, E.; Frühauf, B.; Predel, B. Phase Transformations in Solids; North-Holland: Amsterdam, The Netherland, 1984; Volume 21, pp. 461-466.

43. Poerschke, R.; Wagner, W.; Wollenberger, H. Alloy decomposition in Cu-Ni-Fe. I. Equilibrium states and kinetics of homophase fluctuations. J. Phys. F Met. Phys. 1986, 16, 421-434.

44. Wagner, W.; Poerschke, R.; Wollenberger, H. Alloy decomposition in Cu-Ni-Fe. III. Influence of electron irradiation on decomposition kinetics and morphology. J. Phys. F Met. Phys. 1987, 17, 2017-2029.

45. Turchanin, M.A.; Agraval, P.G.; Abdulov, A.R. Phase equilibria and thermodynamics of binary copper systems with 3D-metals. VI. Copper-nickel system. Powder Metall. Met. Ceram. 2007, 46, 467-477.

46. Wang, W.-H.; Bai, H.Y.; Wang, W.K. Collective interdiffusion in compositionally modulated multilayers. J. Appl. Phys. 1999, 86, 4262-4266.

(C) 2015 by the authors; licensee MDPI, Basel, Switzerland. This article is an open access article distributed under the terms and conditions of the Creative Commons Attribution license (http://creativecommons.org/licenses/by/4.0/). 